\title{
3D Characterization of Porous Copper Thin Films
}

Andi Wijaya ${ }^{1,2}$, Barbara Eichinger ${ }^{3,4}$, Martin Mischitz ${ }^{4}$ and Roland Brunner ${ }^{1 *}$

1. Materials Center Leoben, Forschung GmbH, Leoben, Austria.

2. Montanuniversität Leoben, Leoben, Austria.

3. University of Graz, Graz, Austria.

4. Infineon Technologies Austria AG, Villach, Austria.

* Corresponding author: r.brunner@mcl.at

The interest in porous materials has gained lots of attraction in recent years because of their possible applications e.g. for catalyst [1], in biomimetic [2], or solar cells [3]. In general, the functionality of porous materials is given by their microstructure. Therefore, porous metals are also interesting for future applications in microelectronics. A possible application would be to use porous thin films as a metallization within the semiconductor offering tunable elastic, thermal and electrical properties. With smaller elastic modulus, the metallization layer would exert smaller stress on the device. Another benefit is the increase of specific surface area, therefore thermal management of the device would be better.

In this paper we performed 3D characterization of porous copper thin films by using a scanning electron /focused ion beam (SEM/FIB) microscope from Zeiss (AURIGA ${ }^{\circledR}$ - CrossBeam workstation). Therefore, we set up a pre-processing routine for artefact reduction especially to deal with curtain, shadowing and shine through artefacts and present an automatic image-processing method that enhances the detection of the pores without the need of any preceding infiltration procedure of the samples. Our new method show a high precision ( $>93.4 \%$ in average) and a high recall rate ( $>91.3 \%$ in average) with respect to manual segmentation.

We studied porosity, pore size distributions, pore orientations, and tortuosity from the segmented data with several techniques e.g. watershed, skeletonization, Feret diameters, and tortuosity. In addition, we performed finite element simulations on the segmented data for the prediction of effective mechanical, thermal and electrical properties. We could see that the elastic moduli, thermal and electrical conductivity highly depended on the microstructural properties [4]. The combination of 3D characterization and efficient image analysis techniques is highly interesting for enhanced design guidelines as well as for an improved feedback to the production [5].

\section{References:}

[1] CMA Parlett, K Wilson and AF Lee, Chemical Society Reviews 42 (2013), p. 3876.

[2] E Vennat et al., Dental Materials 31(3) (2015), p. 225

[3] WA Badawy, Journal of Advanced Research Bd. 6 (2015), p. 123.

[4] M Ashby et al., in "Metal Foams: A Design Guide", (Butterworth Heinemann, Oxford).

[5] Financial support from FFG, Produktion der Zukunft (No. 853467 "ProQualiKu") and by the Austrian Federal Government (in particular from Bundesministerium für Verkehr, Innovation und Technologie and Bundesministerium für Wissenschaft, Forschung und Wirtschaft) represented by Österreichische Forschungsförderungsgesellschaft $\mathrm{mbH}$ and the Styrian and the Tyrolean Provincial Government, represented by Steirische Wirtschaftsförderungsgesellschaft $\mathrm{mbH}$ and Standortagentur Tirol, within the framework of the COMET Funding Programme (No. 837900) is also gratefully 
acknowledged.

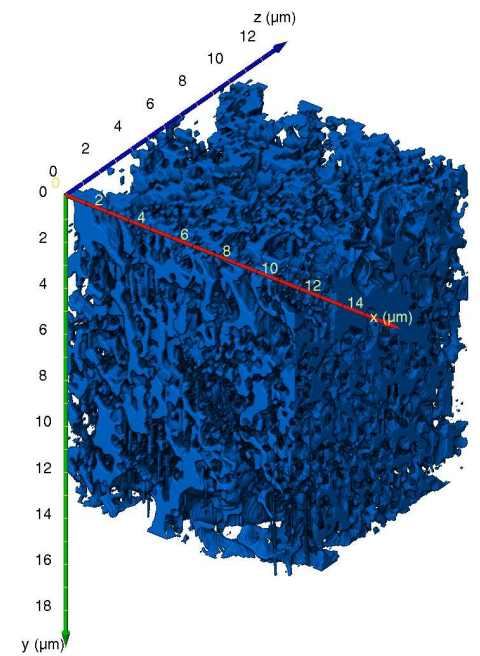

(a)

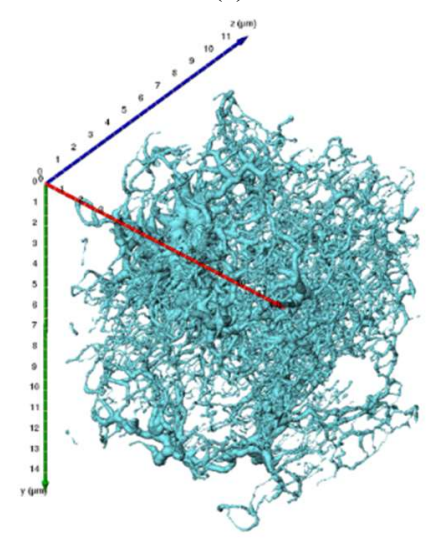

(c)

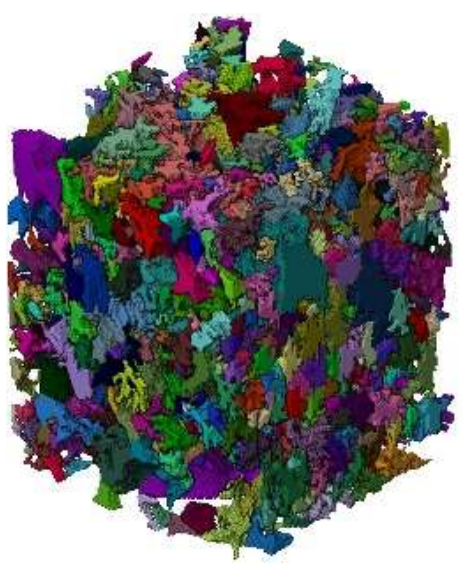

(b)

Conductivity

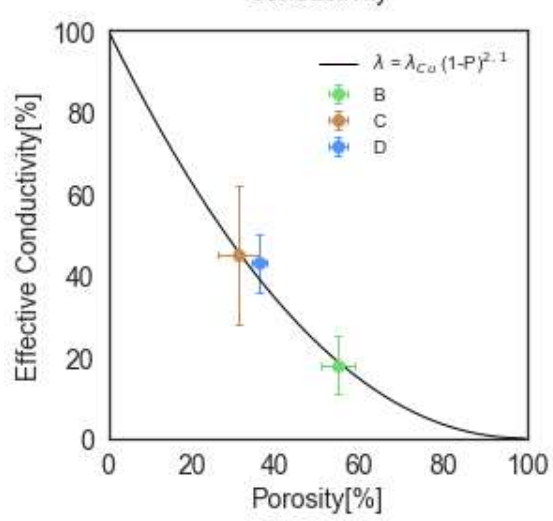

(d)

Figure 1. (a) FIB-nt image (grayscale) and the pore network segmentation (blue). (b) Watershed segmentation of pore network. (c) Skeletonization operation of the pore network. (d) Conductivity as a function of the porosity samples from FEM and the scaling laws of metal foams [4]. 